\title{
A não percepção da obesidade pode ser um obstáculo no papel das mães de cuidar de seus filhos
}

\author{
The non perception of obesity can be an obstacle \\ to the role of mothers in taking care of their children
}

${ }^{1}$ Departamento de Pediatria, Faculdade de Ciências Médicas,

Universidade Estadual de Campinas (Unicamp). R. Tessália Vieira de Camargo 125, Cidade Universitária. 13083-970 Campinas SP. abarros@fcm.unicamp.br ${ }^{2}$ Departamento de Psicologia Médica e Psiquiatria, Faculdade de Ciências Médicas, Universidade Estadual de Campinas (Unicamp), Campinas, $\mathrm{SP}$.

\begin{abstract}
The goal of this study is to review articles in the scientific literature investigating the role of mothers, fathers and relatives in the treatment of obesity of their children. A search for original articles was conducted in the BIREME (BVS), PUBMED (MEDLINE) and Scielo platforms from 2004 to 2010, using the key words "the role of parents" and "childhood obesity," "the role of parents" and "child obesity treatment," "the mother's role" and "childhood obesity," "the mothers role" and "child obesity treatment." Twenty papers were selected and divided into two thematic categories. It was revealed that there are implications of the role of family, namely the father or mother, on the obesity of children, and more specifically related to the role of mothers. The conclusion drawn is that the role of parents and relatives is fundamental in promoting healthy behavior and lifestyles. Distorted maternal perception leads to excess weight being perceived as normal and this may prejudice treatment of child obesity. Acknowledging that the children are overweight most probably promotes greater acceptance of the need for treatment, in addition to being the necessary impetus for seeking professional assistance.
\end{abstract}

Key words Review of the literature, Obesity in childhood and adolescence, the role of mothers
Resumo O objetivo deste estudo foi revisar na literatura científica os artigos que investigam o papel das mães, dos pais e das famílias no tratamento da obesidade dos filhos. Buscou-se artigos originais nas plataformas Bireme (BVS) e Pubmed (Medline) e na base de dados Scielo, entre os anos de 2004 a 2010, com os descritores "the role of parents" and "childhood obesity", "the role of parents" and "child obesity treatment", "the mothers role" and "childhood obesity"; "the mothers" role" and "child obesity treatment", e em português. Foram selecionados 20 estudos, divididos em duas categorias temáticas. Verificou-se que há implicações do papel da familia, pai ou mãe na obesidade das crianças e mais especificamente, dos fatores relacionados ao papel das mães. Identificou-se como fundamental o papel dos pais, mães e familiares no sentido de promover comportamentos e estilos de vida saudável. A percepção materna distorcida faz com que o excesso de peso seja percebido como normal e isso pode prejudicar o tratamento. O reconhecimento do excesso de peso das crianças possivelmente poderá promover uma melhora na adesão e, além disso, pode ser o requisito necessário para a procura de ajuda profissional.

Palavras-chave Revisão da literatura, Obesidade na infância e adolescência, Papel das mães 


\section{Introdução}

As teorias ambientalistas explicam as mudanças nos hábitos atuais de vida da nossa sociedade, práticas como abandono do aleitamento materno, a utilização de alimentos formulados e processados, difusão de jogos eletrônicos e a prática de assistir televisão por muitas horas, em detrimento das atividades físicas como brincadeiras de rua, andar de bicicleta, caminhar pelo bairro, apresentam-se como fatores que contribuíram enormemente para as mudanças do estilo de vida e são considerados determinantes para o aumento da obesidade na infância ${ }^{1,2}$.

Nestle assegura que a obesidade é fruto sociológico da globalização, que favoreceu a chamada transição nutricional com a "cocacolonização" e os "junk-foods". De um lado, os avanços tecnológicos permitiram melhorias na agricultura evitando a fome em massa, mas, de outro, não impediram a ascensão do consumo generalizado dos alimentos com baixo valor nutritivo ${ }^{3}$.

Antes da década de 90 a obesidade na infância não era considerada um problema, pois haviam casos isolados ou associados a doenças genéticas como a Síndrome de Prader-Willi. No inicio dos anos noventa revelou-se outra situação, a obesidade no adulto tornou-se epidêmica e novas estratégias começaram a ser desenvolvidas para o estudo sistemático e o desenvolvimento de critérios para definir a obesidade infantil e a implementação de sistemas de prevenção, pois também na infância e na adolescência a crescente prevalência de obesidade era considerada uma epidemia ${ }^{4}$.

É consenso considerar a multiplicidade de fatores que estão associados à obesidade, nos quais a genética, o metabolismo e o ambiente interagem e refletem em diferentes casos clínicos. Para Barros Filho, as condições ambientais são as que têm exercido grande influência para o aumento da obesidade, e se o ambiente não mudar, dificilmente o obeso conseguirá emagrecer e manter-se magro. As mudanças da sociedade, ou seja, o ambiente como um todo, as famílias e o ambiente familiar devem provocar estímulos de mudanças nos hábitos e estilo de vida das pessoas ${ }^{5}$.

Estudos atuais discutem a importância do papel das mães e das famílias como fator fundamental para o sucesso no tratamento da obesidade de crianças e de adolescentes considerando-se que nessa faixa etária os indivíduos são dependentes de seus pais e/ou cuidadores. As crianças estão subordinadas às condições familiares, como a condição socioeconômica e cultural e sofrem influência das consequências dos conflitos no ambiente familiar, como as desavenças conjugais, violência familiar, doenças da mãe ou de algum membro da família, acesso direto e ilimitado às tecnologias como computadores, videogames e celulares. Resultando em fatores que predispõem toda a familia à obesidade e originando o que se chama de família obesogênica ${ }^{6}$.

Alguns estudos apontam para conclusões de que as taxas de obesidade infantil estão associadas com a obesidade materna, indicando a relação nessa diáde mãe-filho como fator de risco para a obesidade infantil ${ }^{6,7}$.

A obesidade entre os familiares é um fator de risco preponderante para a obesidade na infância, pois o risco de uma criança ser obesa aumenta em função da obesidade dos pais ${ }^{6}$. Segundo Novaes et al. o papel dos pais é fator fundamental de mudança, desse modo o foco deve ser na diminuição do peso dos pais, principalmente das mães, a conscientização de que isto se relaciona aos hábitos alimentares da familia e de que essas práticas alimentares inadequadas podem contribuir para a permanência da obesidade nas crianças. Além disso, estima-se que a redução do sobrepeso dos pais, principalmente das mães, melhora o comportamento alimentar e pode repercutir no hábito alimentar da família como um todo com consequências no estado nutricional das crianças ${ }^{6,8}$.

Desse modo o objetivo deste estudo foi analisar a relação do papel da mãe, dos pais ou cuidadores e o tratamento da obesidade de crianças e adolescentes, na busca de uma melhor compreensão dos papéis familiares nos cuidados com a alimentação das crianças e, principalmente, no tratamento da obesidade.

\section{Método}

Realizou-se uma revisão da literatura de artigos científicos, inicialmente no período de cinco anos e posteriormente estendida até o final de 2010, ou seja, de 2004-2010. A consulta deu-se nas plataformas Bireme (BVS), Pubmed (Medline) e na base de dados Scielo, com os descritores: "the role of parents" and "obesity childhood", "the role of parents" and "child obesity treatment", "the mothers role" and "obesity childhood"; "the mothers" role" and "child obesity treatment", e seus equivalentes em português.

O método adotado foi a pesquisa bibliográfica a fim de delimitar o tema do estudo, no qual buscou-se selecionar artigos que pesquisaram o mesmo fenômeno. 
Os critérios de inclusão foram: 1. Tempo: 2004-2010; 2. Público alvo: mães, pais e famílias de crianças e adolescentes obesos; 3. Idiomas: inglês e português; 4 . Tipo de estudo: qualitativos e quantitativos. Os critérios de exclusão foram os artigos de revisão e os trabalhos que não estavam alinhados com o objeto pesquisado e não se enquadravam nos ítens acima descritos.

Os dados coletados foram lidos de acordo com as fases: exploratória, seletiva, analítica e interpretativa. Esse processo conduziu ao relacionamento dos dados com o objetivo proposto, e assim conferiu um significado mais amplo do material coletado. A pesquisa analisou os estudos que enfocaram a relação entre o papel das mães, dos pais e/ou dos familares e a obesidade na infância e na adolescência (Figura 1).

\section{Resultados}

Foram encontradas 48 referências, das quais analisou-se tipo de pesquisa, objetivos e resultados. Após essa etapa selecionou-se 20 trabalhos, e foram excluídos da amostra final: artigos que não estavam em concordância com o objetivo da busca e artigos de revisão. Realizou-se a análise temática dos estudos selecionados, os quais foram agrupados em duas categorias. Para a apresentação construiu-se uma tabela para cada categoria temática, e posteriomente os dados foram discutidos.

\section{Síntese dos dados e discussão}

Os dados foram analisados qualitativamente. Foram lidos 20 artigos os quais foram selecionados em dois temas: a) o papel dos pais, das mães e dos familiares relacionado à obesidade de crianças e adolescentes e b) a percepção das mães, dos pais e das familias a respeito do peso de seus filhos e sua relação com a obesidade. Sendo que o segundo tema apresentou-se como um desdobramento do primeiro.

\section{O papel das mães, dos pais e da familia relacionado à obesidade de crianças e adolescentes}

Do total dos estudos analisados foi percebido como preponderante o enfoque nas mães, como cuidadora principal, e a relação do seu papel com o excesso de peso e/ou obesidade de seus filhos, ou seja, de 20 artigos analisados 13 estu-

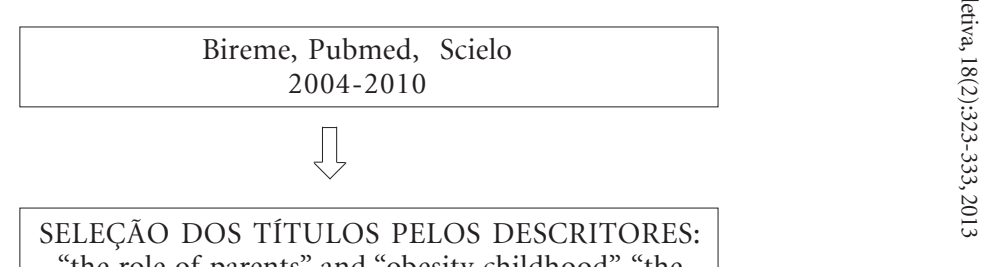

"the role of parents" and "obesity childhood", "the role of parents" and "child obesity treatment", "the mothers' role" and "obesity childhood"; "the mothers" role" and "child obesity treatment" 48 artigos

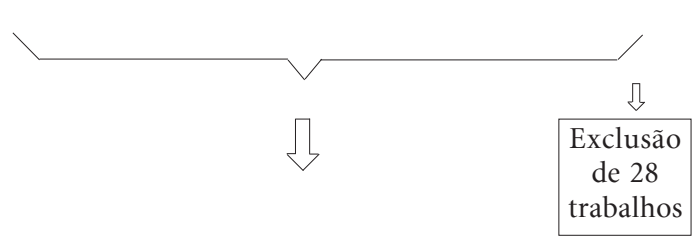

\begin{tabular}{|c|c|}
\hline \multicolumn{2}{|c|}{$\begin{array}{c}\text { Pré-seleção após a leitura dos resumos } \\
20 \text { artigos }\end{array}$} \\
\hline $\begin{array}{c}\text { Estudos que enfatizam o } \\
\text { Papel da Mãe, dos pais e da } \\
\text { família como fator } \\
\begin{array}{c}\text { fundamental no tratamento } \\
\text { da obesidade de crianças e } \\
\text { adolescentes } 11 \text { artigos. }\end{array}\end{array} \begin{array}{c}\text { Estudos que } \\
\text { analisaram a } \\
\text { percepção das mães, } \\
\text { dos pais e das famílias } \\
\text { a respeito do peso de } \\
\text { seus filhos: 09 artigos. }\end{array}$ \\
\hline
\end{tabular}

Figura 1. Fluxograma: seleção dos artigos.

dos pretenderam este enfoque, quatro trabalhos destacaram o papel de pais e de mãe e dois focalizaram as famílias (Quadro 1).

O papel dos pais, seus comportamentos alimentares e estilo de vida, associados a maiores riscos de sobrepeso e obesidade na infância foi investigado no estudo longitudinal de Wake et al. ${ }^{13}$. Em uma amostra composta por 4.983 crianças com idade entre 4-5 anos, 15\% estavam com sobrepeso e $5 \%$ obesas (International Obesity Task Force), as conclusões reforçaram a hipótese da influência do ambiente em detrimento dos aspectos genéticos da obesidade, pois os comportamentos parentais foram associados a maiores riscos e, ainda, pode-se verificar o impacto na fase posterior do desenvolvimento, que é quando as crianças adquirem mais autonomia em relação ao controle da alimentação e da atividade física ${ }^{13}$. Essa pesquisa corrobora a importância do envolvimento dos pais e de ações co- 


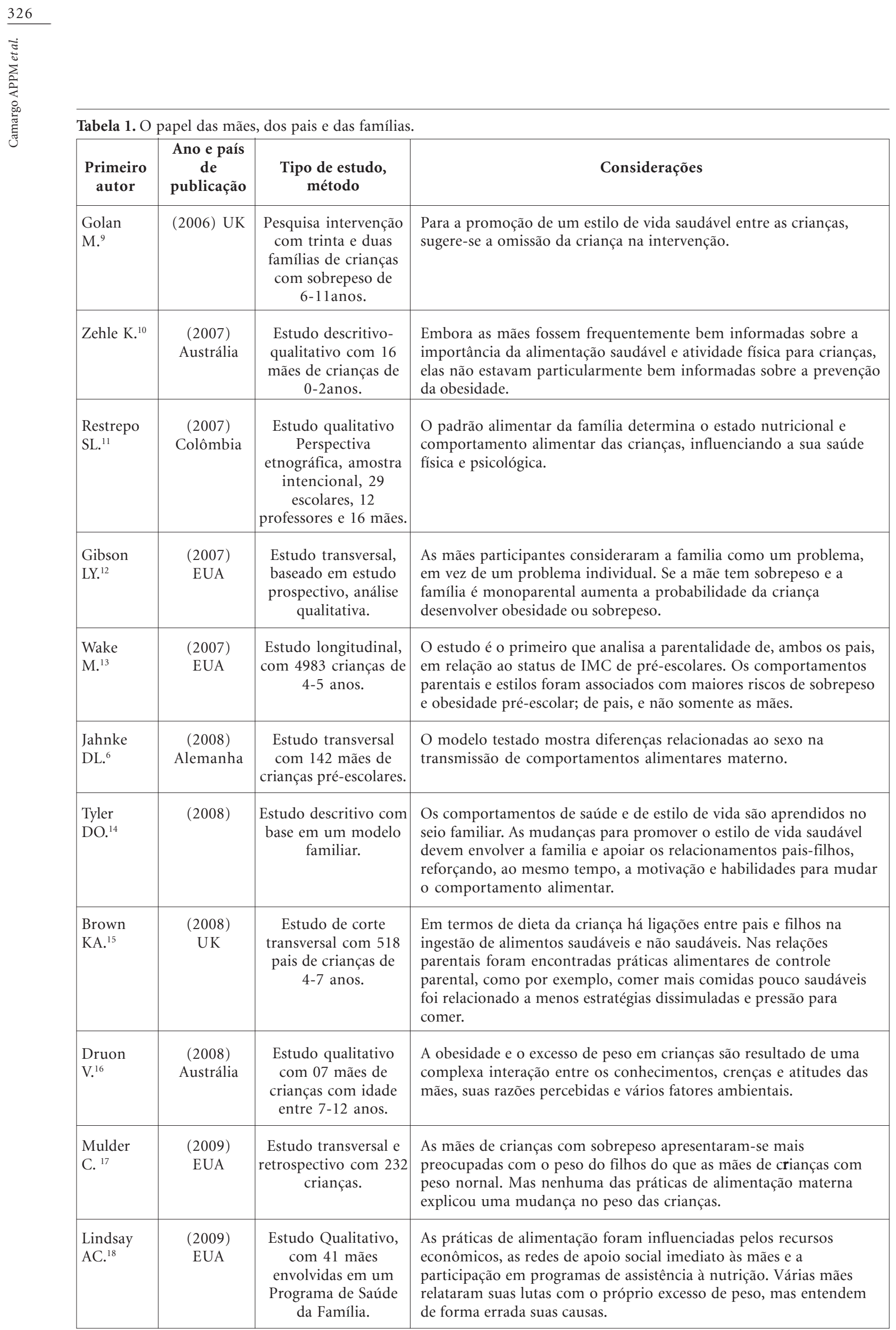


munitárias, que objetivem a melhoria dos atributos parentais.

A respeito da familia influenciando os comportamentos de saúde e estilo de vida de crianças e adolescentes, destacam-se os estudos de Restrepo $^{11}$ e Tyler e Horner ${ }^{14}$.

Restrepo descreveu as dinâmicas presentes na alimentação e nutrição de crianças em idade escolar, do ponto de vista dos próprios escolares, dos professores e dos familiares. Por meio de uma amostra intencional composta por: a) crianças divididas em três grupos: com déficit nutricional, com peso adequado e com excesso de peso; b) dos familiares convocados participaram 16 mães, 01 pai e uma irmã; e c) 12 docentes e 01 pessoa encarregada pela merenda escolar, este estudo etnográfico pôde demonstrar que as práticas alimentares utilizadas pelos pais tem fundamental importância nos habitos de alimentação das crianças. Além disso, fator acrescido por este estudo, problemas relacionados à desnutrição e à obesidade também apresentam-se no contexto escolar, e, assim, devem ser abordados pela comunidade por meio de práticas educativas ${ }^{11}$.

O estudo de Restrepo sugere que as estratégias para analisar as mudanças alimentares deveriam estabelecer pontes entre as pesquisas quantitativas e qualitativas, a fim de complementar as compreensões do fenômeno da obesidade, gerando melhores ferramentas de intervenção ${ }^{11}$.

A partir de um estudo de intervenção com pais e filhos, os autores descrevem um modelo de abordagem centrada na familia, e consideram como imperativo aquelas baseadas nos familiares, pois comportamentos e estilos de vida são aprendidos no seio familiar, e as intervenções que envolvem pais-filhos focando nas relações de apoio podem motivar e desenvolver habilidades de mudança no comportamento alimentar. Essas estratégias, dirigidas para os cuidados primários de saúde, podem desenvolver uma parceria de colaboração com as famílias ao invés de desenvolver práticas normativas ${ }^{14}$.

Ambos os estudos acima citados aprovam a hipótese de que as intervenções devem promover mudanças no estilo de vida das familias e apoiarem os relacionamentos pais-filhos.

Os comportamentos dos pais e não somente das mães, são identificados como fator de maior risco para o sobrepeso e da obesidade em crianças em idade pré-escolar ${ }^{10,13,15}$. Há um impacto das práticas alimentares dos pais na dieta das crianças, principalmente no que diz respeito à ingestão de alimentos considerados não saudáveis.
De acordo com 13 estudos aqui selecionados, o enfoque na mãe, como cuidadora principal, pressupõem que é ela quem se responsabiliza pelos cuidados de alimentação, higiene e educação dos filhos. Um dado interessante e levantado por Zehle et al. é que apesar das mães estarem bem informadas a respeito de alimentação saudavel, elas não demonstram estar particularmente bem informadas a respeito da prevenção da obesidade, o que possivelmente, pode resultar em comportamentos errôneos no que diz respeito à nutrição e à atividade física ${ }^{10}$.

O estudo acima, ainda identificou que algumas das práticas de controle parental utilizam alimentos não saudáveis, o que prejudica a alimentação adequada e pode levar à preferência por esses tipos de alimentos ${ }^{10}$.

No estudo de Zehle et al. as mães não perceberam seu comportamento sedentário modelando o comportamento para a atividade física dos filhos. Os autores concluíram que isso possivelmente aumenta a complexidade do problema da obesidade na infância e dão um alerta para os programas de prevenção a se concentrarem na prevenção da obesidade dos pais ${ }^{10}$.

A respeito dos programas de prevenção, o estudo acima indicou uma possivel ineficácia desses serviços e a importância da adequada influência dos enfermeiros quando se trabalha com prevenção de obesidade da criança, defendendo uma abordagem intervencionista ao invés de práticas informativas. Dando um alerta para que os programas se concentrem em desenvolver competências necessárias aos pais, para que se possa estabelecer e desenvolver bases seguras de hábitos alimentares e de atividades físicas saudáveis.

No estudo qualitativo de Druon et al. ${ }^{16}$ corroborou-se que embora as mães tenham conhecimentos a respeito de um estilo de vida mais saudável elas encontram muitas barreiras na implementação de mudanças tanto na dieta quanto na atividade física. E essas barreiras vão desde crenças, como causar danos à saúde e à autoestima dos filhos, a atitudes a respeito da obesidade, quanto à dificuldade das mães lidarem com o próprio excesso de peso. As mães que reconhecem e se apresentam preocupadas com o sobrepeso dos filhos, mas que não demonstram na prática a mudança na alimentação, resultam em não melhora do controle de peso ${ }^{17}$.

Um fator que pode prejudicar as práticas terapêuticas em obesidade na infância é uma percepção alterada da mãe do estado nutricional do filho, pois os pais podem estar predispostos a 
não aderirem às propostas. No Canadá um estudo realizado em $2007^{13}$ concluiu que grande parte daquela amostra de pais não reconheceu que seus filhos estavam acima do peso ou com obesidade, ainda, 22\% classificaram como baixo peso as crianças que apresentavam peso normal e $63 \%$ como excesso de peso crianças com obesidade. $\mathrm{O}$ estudo sugeriu que estratégias de saúde pública para aumentar a consciência dos pais do estado de peso de seus filhos pode ser o primeiro passo no esforço para prevenir a obesidade na infância.

As mães podem considerar a familia como um problema, em vez de identificar isso como um problema individual ${ }^{12}$. Se de um lado a sociedade age como um sistema que provoca mudanças na vida das pessoas, as familias também têm o papel no desenvolvimento e na promoção da saúde das crianças, por esse motivo as práticas terapêuticas devem envolver a familia e apoiar o relacionamento pais-filhos, e não somente focar na criança ou na relação mãe-filho ${ }^{13,15}$.

As implicações do papel da familia, mãe ou pai, na obesidade dos filhos pode estar relacionada com alguns fatores tais como crenças pessoais, familiares e culturais. Os valores culturais podem apresentar-se como obstáculos para a adoção de comportamentos mais saudáveis ${ }^{19}$. $\mathrm{E}$ a dificuldade em reconhecer e perceber o estado corporal das crianças também pode estar relacionado com essas crenças.

Estudo realizado por Golan e Kaufman ${ }^{9}$ sugeriu que se omita as crianças da participação ativa nas estratégias de intervenção, considerando que pode ser benéfico uma orientação voltada somente aos pais, no sentido de promover um estilo de vida mais saudável e, consequentemente, a redução de peso, já que os comportamentos de saúde e o estilo de vida são aprendidos no seio familiar.

\section{A percepção das mães, dos pais e das famílias a respeito do peso dos filhos e sua relação com a obesidade infantil}

Um bom indicador seria a mãe perceber que o peso corporal pode estar relacionado com a saúde da sua criança, e esse reconhecimento implicaria numa melhora no papel da mãe com os cuidados com a alimentação dos filhos. O Quadro 2 apresenta os estudos que analisaram a percepção materna e sua relação com o excesso de peso ou a obesidade dos filhos.

Em cinco, dos oito artigos analisados, identificou-se a não percepção das mães, ou dos pais, a respeito do "status" corporal em relação ao que atualmente é considerado pelas áreas de saúde como adequado. Tanto há dificuldade de reconhecer o sobrepeso ou a obesidade das crianças, quanto há uma tendência a minimizar a imagem corporal, relatando uma criança mais leve do que ela é realmente ${ }^{20-24}$.

No estudo qualitativo de Crawford et al..$^{20}$ que objetivou avaliar as crenças maternas de mães latinas por meio de questionário semiestruturado e grupo focal, com quarenta e três mães e avós de crianças de 2-5 anos, a análise desse material revelou temas emergentes que foram categorizados em quatro áreas funcionais: 1 . crenças em torno do peso; 2. o impacto do sobrepeso; 3 . valores e preocupações; 4 . estratégias para mudanças dos padrões alimentares. Os resultados revelaram que as crenças culturais podem ser um obstáculo para o sucesso da prevenção e do tratamento do sobrepeso, e os autores sugeriram que entre as crenças maternas, a respeito da saúde dos filhos, um moderado excesso de peso não era visto como problema.

Além disso, as mães que participaram da pesquisa relataram acreditar que um peso extra ajuda as crianças a se recuperarem melhor de doenças e que a magreza está associada com problemas de saúde. Esses valores culturais foram identificados como barreiras para a adoção de comportamentos saudáveis, mas um elemento chave entre os problemas encontrados neste estudo foi a dificuldade das mães de reconhecer o excesso de peso dos filhos e a percepção da relação peso e saúde que foi pouco associada por elas. Esse trabalho forneceu evidências consistentes de que é necessário um novo quadro de educação nutricional com mães latinas. Os autores alertam que o rápido aumento da prevalência de sobrepeso na infância tende a torna-lo uma norma ${ }^{19}$.

Em concordância com outros estudos analisados aqui nesta revisão $0^{14,18,23,24}$, a pesquisa acima corrobourou a importância do envolvimento da familia, e principalmente das mães, na prevenção do sobrepeso, ressaltando que é pouco provável acontecer a mudança no comportamento de crianças sem o apoio de familiares.

A relação percepção materna e sobrepeso e obesidade de filhos foi examinada em um estudo transversal, realizado em Buenos Aires, em que foram avaliadas 321 mães de crianças pré-escolares, com idade média de 4,39 anos e de ambos os sexos. A análise a respeito da percepção materna identificou uma diferença significativa na distorção da percepção da imagem corporal entre as mães de crianças com peso normal (17\%) e mães de crianças com sobrepeso ou risco de sobrepeso 
Quadro 2. A percepção materna.

\begin{tabular}{|c|c|c|c|}
\hline $\begin{array}{l}\text { Primeiro } \\
\text { autor }\end{array}$ & \begin{tabular}{|c|} 
Ano e \\
país de \\
publicação
\end{tabular} & $\begin{array}{l}\text { Tipo de estudo, } \\
\text { método }\end{array}$ & Considerações \\
\hline $\begin{array}{l}\text { Hirschler } \\
\mathrm{V}^{19}\end{array}$ & $\begin{array}{c}(2006) \\
\text { Argentina }\end{array}$ & $\begin{array}{l}\text { Estudo transversal, com } \\
321 \text { mães de pré- } \\
\text { escolares, por } \\
\text { questionarios. }\end{array}$ & $\begin{array}{l}\text { A percepção de mães sobre a imagem corporal e os hábitos } \\
\text { alimentares dos filhos apresentou-se como um preditor de sobrepeso. }\end{array}$ \\
\hline $\begin{array}{l}\text { Crawford } \\
\text { PB. }^{20}\end{array}$ & $\begin{array}{l}(2004) \\
\text { EUA }\end{array}$ & $\begin{array}{l}\text { Pesquisa de campo com } \\
\text { a utilização de grupos } \\
\text { focais e entrevistas } \\
\text { semiestruturadas, com } \\
43 \text { mães. }\end{array}$ & $\begin{array}{l}\text { Identificou-se que as mães tinham dificuldade em reconhecer o } \\
\text { sobrepeso de sua criança e também sua percepção de saúde não } \\
\text { estava ligada ao peso corporal. Certos valores culturais foram } \\
\text { percebidos como obstáculos à adoção de comportamentos saudáveis. }\end{array}$ \\
\hline Murnan $\mathrm{J}^{25}$ & $\begin{array}{l}(2006) \\
\text { EUA }\end{array}$ & $\begin{array}{l}\text { Estudo realizado por } \\
\text { meio de } 700 \\
\text { questionários enviados } \\
\text { a pais de crianças de } \\
\text { escola primária em } \\
\text { Ohio. }\end{array}$ & $\begin{array}{l}\text { Os resultados deste estudo sugerem que os pais de Ohio são } \\
\text { favoráveis às intervenções de base escolar com foco na alimentação } \\
\text { saudável e atividade física. Além disso, acreditam que o ambiente } \\
\text { escolar deve ajudar a reduzir a prevalência de sobrepeso em crianças. }\end{array}$ \\
\hline Hackie $M .{ }^{26}$ & $\begin{array}{l}(2007) \\
\text { EUA }\end{array}$ & $\begin{array}{l}\text { Estudo descritivo por } \\
\text { replicação e } \\
\text { comparativo a uma } \\
\text { variável demográfica. }\end{array}$ & $\begin{array}{l}\text { Mães hispânicas de crianças com sobrepeso podem não perceber as } \\
\text { suas crianças com sobrepeso. A intervenção da enfermagem não } \\
\text { pode ser eficaz se presume que a mãe reconhece o peso do filho } \\
\text { como um problema. Enfermeiros devem estar conscientes do } \\
\text { impacto das crenças culturais sobre o sobrepeso na infância, e fazer } \\
\text { ajustes em sua abordagem educativa. }\end{array}$ \\
\hline $\begin{array}{l}\text { Boa-Sorte } \\
\mathrm{N} \cdot{ }^{27}\end{array}$ & $\begin{array}{l}(2007) \\
\text { Brasil }\end{array}$ & $\begin{array}{l}\text { Estudo transversal com } \\
1.741 \text { estudantes, e seus } \\
\text { pais, com idades de 6- } \\
19 \text { anos classificados de } \\
\text { acordo com o IMC. }\end{array}$ & $\begin{array}{l}\text { A falta de percepção adequada do peso ocorreu com muita } \\
\text { frequência entre as crianças e suas genitoras, em especial quando } \\
\text { havia excesso de peso, fatores que podem representar obstáculos ao } \\
\text { correto reconhecimento de alterações nutricionais. }\end{array}$ \\
\hline $\mathrm{He} \mathrm{M}^{21}$ & $\begin{array}{l}(2007) \\
\text { Canadá }\end{array}$ & $\begin{array}{l}\text { Estudo transversal com } \\
\text { amostra de conveniênia } \\
\text { com questionário } \\
\text { autoadministrado em } \\
\text { sete escolas primárias, } \\
\text { com crianças de } 4-6 \text { e } \\
\text { seus pais. }\end{array}$ & $\begin{array}{l}46 \% \text { das respostas indicaram uma diferença entre estado de peso real } \\
\text { da criança e a percepção dos pais. Fatores como sexo e etnia das } \\
\text { crianças e das mães influenciaram a habilidade de reconhecer o } \\
\text { estado de peso do filho. Os equívocos dos pais parecia não estar } \\
\text { relacionado com os níveis de escolaridade, renda familiar, ou a idade } \\
\text { do filho. }\end{array}$ \\
\hline $\begin{array}{l}\text { Oude } \\
\text { HGM. }^{22}\end{array}$ & $\begin{array}{c}(2009) \\
\text { Holanda }\end{array}$ & $\begin{array}{l}\text { Inquérito de base } \\
\text { Populacional, com } \\
\text { questionário } \\
\text { autoadministrado com } \\
1.155 \text { pais de crianças } \\
\text { de } 4-5 \text { anos. }\end{array}$ & $\begin{array}{l}\text { Para todas as categorias de peso, os pais representaram a criança } \\
\text { como leves, em ambos as escalas - verbal e visual. O excesso de peso } \\
\text { foi considerado como peso normal, e a obesidade como normal ou } \\
\text { um pouco pesado demais. A percepção de um peso normal em } \\
\text { crianças, de } 4-5 \text { anos, esteve distorcida neste estudo. }\end{array}$ \\
\hline $\begin{array}{l}\text { Gallagher } \\
\text { MR. }^{23}\end{array}$ & $\begin{array}{c}(2009) \\
\text { EUA }\end{array}$ & $\begin{array}{l}\text { Estudo qualitiativo, por } \\
\text { entrevistas etnográficas } \\
\text { com mães mexicanas }\end{array}$ & $\begin{array}{l}\text { As mães se referem no controle da alimentação, ao invés do uso da } \\
\text { dieta que, na percepção delas, seria uma restrição não necessária com } \\
\text { uma criança que ainda esta em desenvolvimento. }\end{array}$ \\
\hline $\begin{array}{c}\text { Warschburger } \\
\mathrm{P}^{24}\end{array}$ & $\begin{array}{c}(2010) \\
\text { EUA }\end{array}$ & $\begin{array}{l}\text { Estudo quantitativo } \\
\text { através de questionário, } \\
\text { com } 209 \text { mães de filho } \\
\text { 3-6 anos. }\end{array}$ & $\begin{array}{l}\text { Das mães participantes, } 64,5 \% \text { apontaram o excesso de peso de } \\
\text { crianças pré-escolares corretamente. Apenas } 48,8 \% \text { das mães } \\
\text { identificaram as silhuetas sobrepeso associadas a um aumento de } \\
\text { risco para problemas de saúde física e } 38,7 \% \text { identificaram as } \\
\text { silhuetas associadas com um risco aumentado de saúde mental. Mães } \\
\text { com menor escolaridade estavam mais propensas a não classificar o } \\
\text { excesso de peso nas silhuetas e subestimar os problemas de saúde } \\
\text { associados. Para seu próprio filho, apenas } 40,3 \% \text { das mães } \\
\text { escolheram silhuetas que estavam em acordo com o status de peso. }\end{array}$ \\
\hline
\end{tabular}


$(87,5 \%)$. Entre as mães de crianças com sobrepeso $23,7 \%$ acreditavam que seu filho realmente apresentava sobrepeso e apenas 1,6\% dessas mães consideravam que o risco para sobrepeso era real. Entre as mães de crianças com obesidade grave $45 \%$ consideraram que seu peso era normal. As mães de crianças com risco para sobrepeso, ou com sobrepeso, apresentaram uma diferença significativa na distorção da percepção dos hábitos alimentares, em relação a mães de crianças com peso normal (90,8\% contra 36,3\%). Das mães com filhos com sobrepeso, ou com risco de sobrepeso, $84 \%$ pensavam que seus filhos comiam adequadamente, ou pouco. Das mães de crianças com obesidade grave $72 \%$ afirmaram que seus filhos comiam adequadamente. Esse estudo demonstrou que a falta de percepção materna, do sobrepeso de crianças em idade pré-escolar, se associa a um maior risco desse problema nesta faixa etária. E indica que os profissionais de saúde deveriam ser mais agressivos em suas condutas e aconselhar adequadamente as familias.

Em uma pesquisa realizada na Alemanha, a incapacidade da mãe reconhecer a relação excesso de peso ou obesidade e os riscos para a saú$\mathrm{de}^{24}$, foi investigado com 209 mães de crianças de 3-10 anos. Mesmo 64,5\% apontando as silhuetas corretamente - e neste caso avaliou-se silhuetas de outras crianças e não dos próprios filhos - 48\% das mães não identificaram essas silhuetas de sobrepeso, associada ao aumento de riscos para a saúde. Ao avaliar as silhuetas dos seus filhos, as mães subestimaram o peso corporal e, desse modo, somente $40,3 \%$ apontaram as silhuetas corretas. O estudo conclui que as possiveis variáveis de subestimação são: sexo da criança, idade, influências sociodemográficas.

Murnan et al..$^{25}$ examinaram as percepções dos pais, de crianças pré escolares, a respeito do papel da escola na prevenção do sobrepeso na infância. Dos 644 inquéritos 53,4\% foram respondidos, e os resultados indicaram que os pais apóiam o papel significativo da escola na prevenção da obesidade e na educação em saúde. O estudo sugere a idéia de que a escola deve atuar com um papel complementar na prevenção da obesidade na infância. De acordo com esse trabalho as escolas e os pais devem trabalhar de forma colaborativa para favorecer um ambiente saudável no sentido de incentivar prática de atividade física e a ingestão de alimentos saudáveis. Muitos pais relataram ser favoráveis à escola limitar ou proibir o acesso a alimentos e bebidas não saudáveis a crianças do ensino fundamental (5-11anos). Uma das implicações deste estudo é que os pais participantes não estavam aptos para avaliar e informar o IMC dos filhos e isso pode indicar uma não compreensão das informações e/ou como efetivamente realizar as mudanças nos hábitos de seus filhos.

Em 2007, Hackie e Bowles realizaram um estudo comparativo, a outro trabalho proposto no ano 2000, e usaram os mesmos procedimentos, mas com uma variável demográfica. Eles verificaram que $61 \%$ das mães não identificaram seus filhos com sobrepeso contra $35 \%$ da pesquisa anterior. Os autores sugeriram que essa discrepância se deva em parte pelo tamanho da amostra que provavelmente não reflete uma população maior, mas também há fatores culturais que possam ter influenciado esse resultado ${ }^{26}$.

Outro dado discutido neste trabalho ${ }^{26}$ é que a cultura pode influenciar a percepção materna, enquanto em Virgínia há apenas 24\% de hispânicos, em Nevada é estimado que em torno de $75 \%$ da população seja de origem mexicana, dado observado pelo estudo de Hackie e Bowles, e a partir do qual infere-se que as mães latinas incorrem de modo preponderante nessa percepção equivoca$\mathrm{da}^{26}$. Dos problemas indicados por essas mães, controlar a ingestação de alimentos dos filhos pode ser a tarefa mais dificil, dados semelhantes foram encontrados em outros estudos ${ }^{19,21,26}$, como por exemplo, "as crianças devem ter o que necessitam". De acordo com os autores, isso possivelmente acontece, pois os pais atendem a pedidos de alimentos dos filhos de acordo com as crenças e normas associadas à sua cultura alimentar.

Em 2004, o estado de Nevada já possuía 21,2\% da sua população com excesso de peso, e isso pode ter causado uma falta geral de ênfase na saúde daquele estado, refletindo no que foi percebido nesta amostra $^{26}$. Um resultado surpreendente foi que $76 \%$ das participantes relataram estar cientes do risco de crianças obesas desenvolverem problemas cardíacos. Finalizando, essa pesquisa relata que não encontrou na literatura estudos étnicos comparativos a respeito do mesmo tema, a percepção materna da obesidade dos filhos. Os autores deixam claro que pelo tamanho da amostra este estudo não pode ser generalizado, e sugerem que o estudo seja aplicado a uma amostra maior. Ainda a respeito da limitação deste trabalho, os autores notam a limitação do instrumento usado, pois não oferece oportunidades de aprofundamento das informações relacionadas à investigação.

No Brasil, Boa-Sorte et al. ${ }^{27}$ realizaram um "estudo comparativo da percepção materna e da autopercepção de crianças de seu estado nutricional identificando fatores associados a erros na 
percepção”. De acordo com os autores a percepção adequada do estado nutricional das crianças deve ser fator de primeira instância nos tratamentos de obesidade na infância, principalmente pelo fato da construção dos hábitos alimentares darem-se no seio familiar, sofrendo influências significativas do ambiente e das atitudes dos pais. Assim, a adequada percepção tanto da familia quanto da criança, possivelmente, promove a melhor adesão e também ser o requisito para a procura de tratamento. Nesse estudo a percepção equivocada prevaleceu entre as mães de crianças de 6-9 anos, esse resultado corroborou pesquisas anteriores e indicou uma tendência das mães subestimarem o peso de seus filhos ${ }^{19,20,21,26,27}$. Os autores explicam essa situação devido às crenças maternas, como "criança gordinha é saudável" e "o crescimento irá normalizar o peso". Concluem que, tanto para a prevenção quanto no tratamento, é imprescindível o reconhecimento, pelos pais, do excesso de peso de suas crianças. Ainda, propõem uma maior divulgação dos riscos para a saúde que a obesidade na infância acarreta, e que o aumento na conscientização seja imprescindível para o sucesso das intervenções e medidas de controle da obesidade na infância.

Ajudar os pais de crianças pré-escolares a reconhecerem o peso e a estarem cientes dos riscos da obesidade e do excesso de peso pode ser um dos primeiros passos para a promoção de um estilo de vida saudável ${ }^{21}$. Estudo realizado no Canadá confirma que dos pais que não reconheciam que seus filhos estavam com excesso de peso ou obesidade, $22 \%$ classificaram erroneamente seus filhos com peso normal como sendo baixo peso, $63 \%$ classificaram como normais crianças com sobrepeso e $63 \%$ perceberam seus filhos obesos sendo sobrepeso.

Em 2006, na Holanda ${ }^{22}$, realizou-se um estudo populacional para avaliar a percepção do peso e o reconhecimento do excesso de peso, de pais de crianças de 4-5 anos. Do total dos 1.155 questionários, 35\% retornaram. Para todas as categorias de peso, em ambas escalas avaliadas - verbal e visual - os pais reconheceram suas crianças como mais leves. Do total, $75 \%$ das mães de crianças com sobrepeso afirmaram que a criança tinha um peso normal. Nas crianças obesas, 50\% das mães acreditam que a criança tinha um peso normal.

É importante ressaltar, no trabalho acima mencionado, o número de questionários devolvidos - 439 - esse dado preocupa considerando a urgência na prevenção do excesso de peso e no desaceleramento da pandemia da obesidade em crianças e adolescentes ${ }^{22,28,29}$.
A percepção dos pais a respeito do peso corporal do filho é distorcida, conclui o trabalho ${ }^{25}$, a razão do não reconhecimento pelos pais da obesidade dos filhos não fica claramente explicado, mas infere este estudo que isso possa estar relacionado a uma mudança no que se percebe como normal, e assim uma criança com peso normal é vista com o baixo peso. O estudo sugere que isso resulta em uma mudança normativa, ou seja, o padrão de normalidade muda, em decorrência da percepção dos pais; o excesso de peso, que deveria ser percebido como sobrepeso passa ser visto como peso normal. Pais de crianças com peso normal relatam perceberem seus filhos como leves e o sobrepeso é compreendido como normal. Portanto, para uma estratégia de prevenção é necessário a participação dos pais, além do reconhecimento do estado corporal dos filhos, dentro dos critérios médicos, e isso pode ser fundamental para coibir a prevalência da obesidade em crianças e adolescentes.

\section{Conclusões}

A influência do comportamento alimentar parental afeta os hábitos dos filhos, e está associado a maiores riscos de desenvolvimento e mantutenção da obesidade. Ao investigar o papel das famílias - ou dos pais - ficou evidente o estudo do papel da mãe como figura central no que diz respeito ao desenvolvimento da criança e, especificamente, nesse caso, no desenvolvimento de hábitos saudáveis.

O papel dos pais, mães e familiares e sua relação com o excesso de peso e obesidade em crianças e adolescentes é fundamental no sentido de promover comportamentos e estilos de vida saudável ${ }^{9,10,14,15,18,19,24}$, tanto no sentido de prevenção quanto no sentido de reverter esse quadro epidêmico da saúde infantil, que se apresenta atualmente no mundo todo. Notou-se que uma tendência dos pais a minimizarem o estado nutricional e, mais preocupante ainda, não terem consciência dos riscos desse estado, e por esse motivo não promoverem mudanças efetivas na dieta e nos hábitos de vida dos filhos ${ }^{21,22,26,27}$.

O papel materno esteve valorizado, mas sabese que a criança se relaciona e se desenvolve em um ambiente mais amplo, geralmente com pais, irmãos, avós, professores, amigos e sociedade. Desse modo as intervenções devem enfocar o ambiente familiar e atuar de modo colaborativo ${ }^{14}$.

Outro aspecto observado diz respeito ao modo como a mãe percebe o estado corporal de 
seu filho e como isso pode estar relacionado ao excesso de peso. A percepção materna distorcida pode prejudicar os tratamentos e isso pode explicar o quadro atual de compreender o sobrepeso como uma norma ${ }^{21,23}$, prejudicando ações preventivas e influenciar a não constatação da necessidade terapêutica. Desse modo podemos propor a perceção materna do estado corporal como um subitem nessa complexa multifatoriedade do excesso de peso e da obesidade em crianças e adolescentes.

O papel dos pais como implicação no tratamento da obesidade pode se relacionar na medida em que, se as mães não reconhecem seus filhos como obesos não adotam ou investem em um novo padrão de comportamento alimentar. O não reconhecimento passa a ser um obstáculo às estratégias de intervenção ${ }^{30}$.

O reconhecimento do excesso de peso pode ser o requisito necessário para a ajuda profissional. Os pais - e não somente as mães - a família e a sociedade têm um papel fundamental no desenvolvimento global do ser humano, tanto na aquisição de estilo de vida saudável, quanto no comportamento alimentar mais adequado.

Mudanças nas intervenções são necessárias, do ponto de vista das estratégias que podem ser mais colaborativas do que normativas, e também em relação ao foco, que pode deixar de recair - somente - no sujeito e na doença para englobar o ambiente no qual ele está inserido, ou seja, envolver sua familia e a comunidade em que o sujeito vive. Além disso, a redução de peso dos adultos pode impactar no peso das crianças.

Os profissionais de saúde podem promover competências e habilidades nas mães, nos pais, nos familiares ou cuidadores focando a melhora no estilo de vida e no comportamento alimentar.

Um aprofundamento no tema e a utilização de instrumentos de pesquisa que permitam a exploração e a interpretação dessa percepção subestimada fazem-se necessários. Uma mudança na abordagem do objeto de pesquisa pode ampliar a compreensão a respeito dessa problemática, ou seja, ao invés de quantificar, explorar e compreender os modos de percepção das mães.
Estudos que visem uma melhor compreensão da relação obesidade na infância e percepção materna, em interação com os outros fatores já identificados na literatura, possivelmente abrirão novas discussões para o conhecimento científico.

\section{Comentários finais}

O papel da mulher na atualidade não foi discutido neste estudo, mas cabe ressaltar a importância desta consideração. Como vimos, na maioria dos trabalhos aqui analisados, quando se investiga o papel da familia e sua relação com o excesso de peso em crianças e adolescentes, o foco recai sobre a mãe, que é compreendida como a cuidadora principal. Sabe-se que o papel da mulher contemporânea mudou muito, principalmente pelo acúmulo de funções, dentro e fora da familia. E as implicações desse novo papel reincidem sobre o desenvolvimento dos filhos, tanto nos aspectos físicos, como psicológicos. Dessa forma há necessidade de se discutir essa questão levando em conta como equacionar uma educação para uma vida mais saudável e a disponibilidade de tempo da mulher nas condições de vida atuais.

\section{Colaboradores}

APPM Camargo, AA Barros Filho, MARGM Antonio e JS Giglio participaram de todas as etapas do estudo e preparação do manuscrito.

\section{Agradecimentos}

Agradecemos a toda equipe do Ambulatório de Obesidade na Infância e na Adolescência do Hospital das Clínicas (Unicamp) e aos Profesores Dr. Roberto Teixeira Mendes, Dr. Sérgio Arruda Saboya e a Dra. Mariana Zambon, pelos cuidadosos comentários e colaboração com este estudo. 


\section{Referências}

1. Oliveira CL, Fisberg M. Obesidade na infância e adolescência: uma verdadeira epidemia. Arq Bras. Endocrinol Metab 2003; 47(2):107-108.

2. Enes CC, Slater B. Obesidade na adolescência e seus principais fatores determinantes. Rev bras epidemiol 2010; 13(1):163-171

3. Nestle M. Eating made simple. Sci Am 2007; 297(3): 60-69.

4. James WPT. Tendências globais da obesidade infantil - consequências a longo prazo. Obesidade na Infância. Anais Nestlé 2002; 62:1-11.

5. Barros Filho AA. Um quebra-cabeça chamado obesidade. J Pediatr 2004; 80(1):1-3.

6. Jahnke DL, Warschburger PA. Familial transmission of eating behaviors in preschool-aged children. Obesity (Silver Spring) 2008; 16(8):1821-1825.

7. Escrivão MAMS, Oliveira FLC, Taddei JAAC, Lopez FA. Obesidade exógena na infância e na adolescência. J Pediatr 2000; 76(Supl. 3):S305-S310.

8. Novaes JF, Franceschini SCC, Priore SE. Mother's overweight, parent's constant limitation on the foods and frequent snack as risk factors for obesity among children in Brazil. Arch Latinoam Nutr 2008; 58(3):256-264.

9. Golan M, Kaufman V. Childhood obesity treatment: targeting parents exclusively v. parents and children. Br J Nutr 2006; 95(5):1008-1015.

10. Zehle K, Wen LM, Orr N, Rissel C. "It's not an issue at the moment": a qualitative study of mothers about childhood obesity. MCN Am J Matern Child Nurs 2007; 32(1):36-41.

11. Restrepo MSL. Children's perceptions about of the feeding and nutrition. Percepciones frente a la alimentación y nutrición del escolar. Perspect Nutr Hum 2007; 9(1):23-35.

12. Gibson LY, Byrne SM, Davis EA, Blair E, Jacoby P, Zubrick SR. The role of family and maternal factors in childhood obesity. Med J Aust 2007; 186(11): 591-595.

13. Wake M, Nicholson JM, Hardy P, Smith K. Preschooler Obesity and Parenting Styles of Mothers and Fathers: Australian National Population Study. Pediatrics 2007; 120(6):e1520-e1527.

14. Tyler DO, Horner SD. Family-centered collaborative negotiation: a model for facilitating behavior change in primary care. USA. J Am Acad Nurse Pract 2008; 20(4):194-203.

15. Brown KA, Ogden J, Vögele C, Gibson EL. The role of parental control practices in explaining children's diet and BMI. Appetite 2008; 50(2-3):252-259.

16. Druon V, Fraser J, Alexander C. Mothers' knowledge, beliefs and attitudes towards their obese and overweight children living in rural north-west of New South Wales. Aus J Rural Health 2008; 16(6): 387-388.

17. Mulder C, Kain J, Uauy R, Seidell JC. Maternal attitudes and child-feeding practices: relationship with the BMI of Chilean children. Nutr J 2009; 8:37.
18. Lindsay AC, Sussner KM, Kim J, Gortmaker S. The role of parents in preventing childhood obesity. Future Child 2006; 16(1):169-186.

19. Hirschler V, González C, Cemente G, Talgham S, Petticnichio H, Jadzinsky M. Cómo perciben las madres de niños de jardín de infantes a sus hijos con sobrepeso? Arch Argent Pediatr 2006; 104(3):221-226.

20. Crawford PB, Gosliner W, Anderson C, Strode P, Becerra-Jones Y, Samuels S, Carroll AM, Ritchie LD. Counseling Latina mothers of preschool children about weight issues: suggestions for a new framework. J Am Diet Assoc 2004; 104(3):387-394.

21. Meizi H, Evans A. Les parents sont-ils conscients que leurs enfants souffrent de surpoids ou d'obésité? S'en préoccupent-ils? Can Fam Physician 2007; 53: 1493-1499.

22. Oude LHGM, Stolk RP, Sauer PJ. How do parents of 4- to 5-year-old children perceive the weight of their children? Acta Paediatrica 2010; 99(2):263-267.

23. Gallagher MR. Maternal perspectives on lifestyle habits that put children of Mexican descent at risk for obesity. J Spec Pediatr Nurs. 2010; 15(1):16-25.

24. Warschburger P, Kröller K. Maternal perception of weight status and health risks associated with obesity in children. Pediatrics. 2009; 124(1):e60-e68.

25. Murnan J, Price JH, Telljohan SK, DAke JA, Boardley D. Parents' perceptions of curricular issues affecting children's weight in elementary schools. J Sch Health 2006; 76(10):502-511.

26. Hackie M, Bowles CL. Maternal perception of their overweight children. Public Health Nursing 2007; 24(6):538-546.

27. Boa-Sorte N, Neri LA, Leite ME, Brito SM, Meirelles AR, Luduvice FB, Santos JP, Viveiros MR, Ribeiro HC. Maternal perceptions and self-perception of the nutritional status of children and adolescents from private schools. J Pediatr 2007; 83(4): 349-356.

28. Oliveira CL, Fisberg M. Obesidade na infância e adolescência: uma verdadeira epidemia. Arq Bras Endocrinol Metab 2003; 47(2):107-108.

29. Han JC, Lawlar DA, Kimm SYS. Childhood obesity. Lancet 2010; 375:1737-1748.

30. Popkin BM. O mundo esta gordo: modismos, tendências, produtos e políticas que estão engordando a humanidade. Rio de Janeiro: Elsevier; 2009.

Artigo apresentado em 20/04/201

Aprovado em 10/07/2011

Versão final aprovada em 14/11/2011 